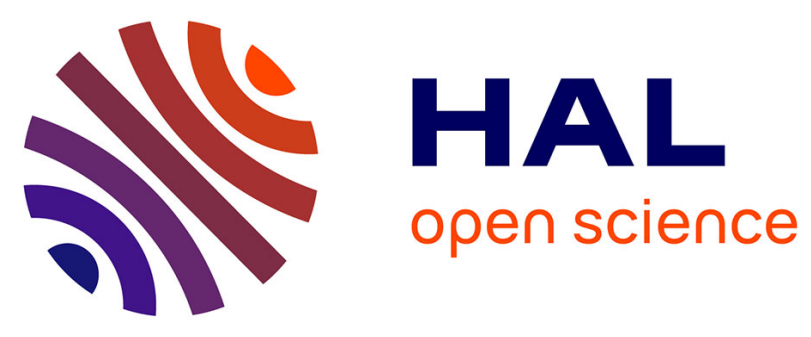

\title{
Exclusive ambulatory management of spontaneous pneumothorax with pigtail catheters, a prospective multicentric study
}

\author{
Alexandre Salé, Laurent Sohier, Marine Campion, Régis Le Hô, Yann Bazin, \\ Cédric Gangloff, Mallorie Kerjouan, Bertrand Delatour, Emmanuel Oger, \\ Stéphane Jouneau
}

\section{To cite this version:}

Alexandre Salé, Laurent Sohier, Marine Campion, Régis Le Hô, Yann Bazin, et al.. Exclusive ambulatory management of spontaneous pneumothorax with pigtail catheters, a prospective multicentric study. Respiratory Medicine, 2020, 166, pp.105931. 10.1016/j.rmed.2020.105931 . hal-02565722

\section{HAL Id: hal-02565722}

\section{https://hal-univ-rennes1.archives-ouvertes.fr/hal-02565722}

Submitted on 14 May 2020

HAL is a multi-disciplinary open access archive for the deposit and dissemination of scientific research documents, whether they are published or not. The documents may come from teaching and research institutions in France or abroad, or from public or private research centers.
L'archive ouverte pluridisciplinaire HAL, est destinée au dépôt et à la diffusion de documents scientifiques de niveau recherche, publiés ou non, émanant des établissements d'enseignement et de recherche français ou étrangers, des laboratoires publics ou privés. 
Exclusive ambulatory management of spontaneous pneumothorax with pigtail catheters, a prospective multicentric study.

Alexandre Salé ${ }^{1}$, Laurent Sohier ${ }^{2}$, Marine Campion ${ }^{3}$, Régis Le Hô ${ }^{4}$, Yann Bazin ${ }^{1}$, Cédric Gangloff ${ }^{5}$, Mallorie Kerjouan ${ }^{1}$, Bertrand Delatour ${ }^{6}$, Emmanuel Oger ${ }^{7}$, Stéphane Jouneau ${ }^{1,8}$

1, Service de pneumologie, centre de compétences pour les maladies pulmonaires rares, Hôpital Pontchaillou, Rennes, France

2, Service de pneumologie, Hôpital du Scorff, Lorient, France

3, Service de cardiologie et maladies vasculaires, Hôpital Pontchaillou, Rennes, France

4, Service d'accueil des urgences, Centre Hospitalier de Vitré, Rennes, France

5, Service d'accueil des urgences, Centre Hospitalier de Vitré, Rennes, France

6, Service de chirurgie thoracique et vasculaire, Hôpital Pontchaillou, Rennes, France

7, Service de pharmacologie, Hôpital Pontchaillou, Rennes, France

8, IRSET UMR 1085, Université de Rennes 1, Rennes, France

All persons who meet authorship criteria are listed as authors, and all authors certify that they have participated sufficiently in the work to take public responsibility for the content, including participation in the concept, design, analysis, writing, or revision of the manuscript. Furthermore, each author certifies that this material or similar material has not been and will not be submitted to or published in any other publication. 


\section{Journal Pre-proof}

Exclusive ambulatory management of spontaneous pneumothorax with pigtail catheters, a prospective multicentric study.

Alexandre Salé ${ }^{1}$, Laurent Sohier ${ }^{2}$, Marine Campion ${ }^{3}$, Régis Le Hô ${ }^{4}$, Yann Bazin ${ }^{1}$, Cédric Gangloff ${ }^{5}$, Mallorie Kerjouan ${ }^{1}$, Bertrand Delatour ${ }^{6}$, Emmanuel Oger ${ }^{7}$, Stéphane Jouneau ${ }^{1,8}$

1, Service de pneumologie, centre de compétences pour les maladies pulmonaires rares, Hôpital Pontchaillou, Rennes, France

2, Service de pneumologie, Hôpital du Scorff, Lorient, France

3, Service de cardiologie et maladies vasculaires, Hôpital Pontchaillou, Rennes, France

4, Service d'accueil des urgences, Centre Hospitalier de Vitré, Rennes, France

5, Service d'accueil des urgences, Centre Hospitalier de Vitré, Rennes, France

6, Service de chirurgie thoracique et vasculaire, Hôpital Pontchaillou, Rennes, France

7, Service de pharmacologie, Hôpital Pontchaillou, Rennes, France

8, IRSET UMR 1085, Université de Rennes 1, Rennes, France 
ABSTRACT

OBJECTIVE: Spontaneous pneumothorax occurs most frequently in young active patients. Published guidelines do not all agree about its initial management; most patients are hospitalised and treated with chest tube. This prospective multicentric cohort study was designed to assess the potential of ambulatory management.

METHODS: We included all consecutive patients with large spontaneous primary (PSP) and secondary pneumothoraces (SSP) presenting at the Lorient, Vitré and Rennes hospitals between December 2013 and July 2016. They were treated with a small-bore pigtail catheter and one-way valve and managed as outpatients following a specific protocol. When this failed, patients were hospitalised on day 4 for suction and surgical pleurodesis was envisaged on day 6. Patients were followed-up for one-year to assess relapse.

RESULTS: Of the 148 patients included (129 PSP, 19 SSP), 122 (82.4\%) were managed exclusively as outpatient with success in $84.5 \%$ of PSP and $68.4 \%$ of SSP patients. There were few complications: 13 vasovagal episodes and 3 minor bleedings. The one-year recurrence rates were $33 \cdot 1 \%$ for PSP and $52 \cdot 6 \%$ for SSP $(\mathrm{p}=$ $0 \cdot 114$ Hazard Ratio = 0.538; IC95\% [0·249-1·161]).

CONCLUSION: These results are consistent with our previous study and confirm that this exclusive ambulatory management of spontaneous pneumothoraces can be successfully implemented in new centres with a high success rate and few complications. 


\section{INTRODUCTION}

Pneumothorax is an abnormal irruption of air into the pleural cavity leading to partial or complete collapse of the underlying lung. Pneumothoraces can be either spontaneous, traumatic or iatrogenic. Primary spontaneous pneumothaces (PSP) occur in patients who have no known underlying lung disease, while those occurring as a complication of a known or suspected lung disease are secondary spontaneous pneumothoraces (SSP). The incidence of PSPs in healthy young adults is $22 \cdot 7 / 100,000$ inhabitants and is more likely to occur in men than in women (ratio: 3/1). ${ }^{1,2}$

Although spontaneous pneumothoraces are common and generally benign, the definitions of large pneumothoraces and their initial management differ. The British Thoracic Society (BTS) and Belgian Society of Pneumology recommend needle aspiration (NA) as a first step, followed by the insertion of chest tube if NA fails ${ }^{3,4}$, whereas the American College of Chest Physicians (ACCP) recommends using small bore pleural catheters. ${ }^{5}$ The European respiratory society (ERS) recently issued a statement recommending NA for the initial management of spontaneous pneumothoraces and ambulatory management if possible, while pointing out the high failure rate of aspiration alone $(25-50 \%) .{ }^{6}$ However, there is little reliable published evidence to help select the best initial management for these patients, as highlighted by Maskell. ${ }^{7}$ Ambulatory management with pigtail catheters is possible. Marquette's team carried out a pilot study ${ }^{8}$ using small bore catheters and later, in 2014, published a study of 48 patient in which 18 were treated with a small bore pigtail catheter connected to a one-way valve and managed as outpatients with a good success rate and few complications..$^{9,10}$ Our Lorient hospital team published a monocentric retrospective study in the same year on 132 patients with PSP or SSP, $78 \%$ of whom were exclusively ambulatory managed. ${ }^{11}$

The present multicentric study is a prospective assessment of our management of a cohort of patients with spontaneous pneumothorax. We implemented the Lorient Hospital management protocol in the Emergency and Pulmonology departments at Rennes and Vitré. Our objectives were to verify the success rate of exclusive ambulatory management of PSP and SSP in other centres (main outcome), to evaluate the safety of the catheter insertion procedure, analgesic consumption, and the one-year recurrence rate (secondary outcomes).

\section{MATERIALS AND METHODS}

\section{Patients}


We included all consecutive patients presenting with a large primary or secondary pneumothorax, aged 15 years or more between December 2013 and July 2016 and who agreed to participate. These patients were treated in the Emergency Departments of Rennes University Hospital, Lorient Hospital, or Vitré Hospital. Traumatic and iatrogenic pneumothoraces were excluded. Signed informed consent was obtained from the older patients $(\geq 18$ years) and signed informed consent from the parents of younger ones. The study was approved by the Rennes Hospital Ethics Committee ( $\left.{ }^{\circ} 14.08\right)$.

Large pneumothoraces were defined according to ACCP criteria (distance from apex to cupola $>3 \mathrm{~cm}$ ) or BTS criteria (presence of a visible rim of air $>2 \mathrm{~cm}$ between the lung margin and the chest wall). ${ }^{3,5}$ Pneumothoraces were diagnosed by the physician in charge, who also established indication of the drainage. Both initial episodes and recurrences were included, even if they had previously been treated by chest tube drainage. Secondary pneumothorax was defined as occurring in patients with known or suspected lung disease. Lung disease was suspected if the patient was over 50 years old and a smoker ( $\geq 10$ pack-year). ${ }^{3,12}$

\section{Study design}

Spontaneous pneumothoraces have been routinely managed using small bore pigtail catheter at the Lorient Hospital since $2007 .{ }^{11}$ Catheters are inserted by one of the hospital respiratory physicians and the patients are followed-up as outpatients by hospital respiratory physicians from Lorient, as set out in the protocol (Figure 1). This protocol is now used routinely in the Rennes (since 2011) and Vitré hospitals (since 2012) (implementation centers).

Patients treated in the Emergency Departments at Rennes or Vitré hospitals had their small bore pigtail catheters inserted by an emergency physician. They were followed-up by respiratory physicians based in the Rennes Hospital out-patients clinic.

The pigtail catheters (8.5-French; $\operatorname{Cook} \odot$, Bloomington, IN) were positioned under local anesthetic (1\% lidocaine) and aseptic conditions in the second or third intercostal space in the midclavicular line. ${ }^{11}$ Catheters were never placed axillarilly as this increased the risk of dislodging it with everyday movements and sweating.

The proper placement of the catheter in the pleural space and the permeability of the system were checked by aspiration (bubbles in the lidocaine syringe). The catheter was then fitted to a one-way mini-Heimlich valve (CCASP-A6ford; Cook $\odot$, Bloomington, IN). The catheter was secured in place with a polyurethane transparent adhesive dressing and a pillow of folded sterile gauze was placed under the catheter to prevent kinking (figure 
1). ${ }^{9,11}$ Complete aspiration through the catheter was not performed before the patient was discharged. The movements of the one-way valve associated with breathing were generally enough to confirm the correct placement of the catheter but its position was also checked by a chest X-ray if the physician in charge so desired. Patients were discharged on day 0 unless the security conditions were not met, again, at the discretion of the physician ${ }^{9}$, at which time they were given information and specific advice. All patients were assessed by respiratory physician every two working days as outpatients. They underwent a clinical examination and catheter aspiration each time; pain, analgesic consumption, and complications were noted. Success was defined as complete or nearly complete lung re-expansion (only a very small rim of apical air) on the chest X-ray before catheter withdrawal. ${ }^{8,9,11}$

Patients were contacted by phone, text message or e-mail 3, 6, 9 and 12 months after inclusion and asked if another pneumothorax confirmed by chest X-ray had occurred and the date of relapse.

\section{Data analysis}

The patient characteristics at inclusion are described first. Means and standard deviations (SD) or medians and extreme values are reported for quantitative variables and the number and percentage of categorical variables. Complications by centre were compared with Chi-squared or Fischer Exact tests, when appropriate.

Recurrence-free survival was calculated from the date of inclusion to the exact date of relapse or the date of last contact (3, 6, 9 and 12 months). Kaplan-Meier curves were used to describe recurrence-free survival and compare recurrence rates in the PSP and SSP groups. Data were analysed using Cox proportional hazards in univariate and multivariate models. In the multivariate models, age, smoking history, body mass index (BMI) and gender were used as adjustment variables. Patients who were lost to follow-up were censored, and patients who underwent bilateral pleurodesis after inclusion were excluded from this analysis. All analyses were done with SAS ${ }^{\circledR}$ (version 9.4) and tested in bilateral formulation, with a statistical significance set at $5 \%$ (alpha error $=0 \cdot 05)$.

\section{RESULTS}

\section{Population characteristics}

We included 148 consecutive patients with large spontaneous pneumothoraces (Lorient $\mathrm{N}=66$, Rennes $\mathrm{N}=59$, Vitré N=23), 129 were PSP and 19 were SSP. The 19 patients with an SSP included 13 with a known underlying lung disease (lung cancer $n=2$, COPD/emphysema $n=11$ ) and 6 with a suspected secondary pneumothorax. Their mean age was 31 years $(\mathrm{SD}=13$; range:16-81), $76 \%$ were smokers or former smokers (mean: 11.8 pack-year; range: 0.5-50 pack-year) (Table 1). One patient with SSP died during follow-up from 
sepsis due to bullae infection not related to the management of his pneumothorax; there were no other deaths by the end of the one-year follow-up.

\section{Main outcome}

Of the 148 patients, $122(82.4 \%,[95 \% \mathrm{CI}=76 \cdot 3 \%$ to $88.6 \%])$ were managed exclusively as outpatients with success on days $2(n=61), 4(n=60)$ or $6(1)$. The ambulatory success rate for patients with PSP was $84 \cdot 5 \%$ $(\mathrm{n}=109)[95 \% \mathrm{CI}=78 \cdot 2-90 \cdot 73 \%]$ and $68 \cdot 4 \%(\mathrm{n}=13)[95 \% \mathrm{CI}=47 \cdot 5-89 \cdot 3 \%]$. One patient could not be admitted on day 4 but his lung was fully re-expanded on day 6; his catheter was removed without hospital admission. Some patients were hospitalized on day 0 and discharged early for personal convenience (eg: one 16 year-old was hospitalized for one night because his mother could only collect him from the hospital the next morning). In total $26(17.5 \%)$ patients were hospitalised, either initially or on day 4, following the protocol (Figure 2). Among SSP, 13 were successfully managed exclusively as outpatient

The mean time in hospital was $6 \cdot 1$ days (1-21 days). The overall success rates on day 4 (including patients who were briefly hospitalised) at the three hospitals did not differ significantly - Lorient (initial centre): $87 \cdot 8 \%$, Rennes and Vitré (implementation centres): 86.6\%.

\section{Secondary outcomes}

\section{Complications}

There were thirteen vaso-vagal episodes during catheter installation and three minor parietal hematomas that did not require surgery or transfusion, and none became infected. Similarly, no pigtail catheter became infected during this study. None of these complications influenced patient management. The rates of complications at the three centres did not differ significantly; eight vaso-vagal episodes and one haemorrhage at Lorient (initial centre) and five + two at Rennes / Vitré (implementation centres).

Analgesics were frequently used; most patients (87.1\%) were given a step 1 analgesic; $27 \cdot 8 \%$ were given step 2 treatment (tramadol or codeine plus acetaminophen) and 9.3\% were given step 3 analgesics (immediate-release morphine sulfate). No injectable analgesics were used in outpatients.

\section{Recurrence-rate}

The overall recurrence rate was 35.6\% after follow-up for one year (Figure 3). Eight patients were lost to followup and censored at the last contact date, and five underwent bilateral pleurodesis after the pneumothorax that led to their inclusion in our study and were excluded from this analysis. The rates of recurrence in the PSP (33.1\%) 
and SSP $(52.6 \%)$ patients one-year post-intervention were not significantly different $(\mathrm{p}=0 \cdot 114$; Hazard Ratio = $0 \cdot 538 ;$ IC95\% [0-249-1·161]).

\section{DISCUSSION}

The success rate of this large prospective study of exclusive ambulatory management of spontaneous pneumothoraces using pigtail catheters and one-way valves was $82.4 \%$ for all pneumothoraces. It was accompanied by few complications and no severe adverse event.

These data are consistent with our previous retrospective monocentric study ${ }^{11}$ in which the success rate was $78 \%$ for those managed exclusively as outpatients. They are also consistent with those quoted in a fairly recent systematic review. $^{13}$

Our previous study was criticised for its retrospective design. ${ }^{14}$ The findings of the present prospective multicentric study confirm those results and also show that this pigtail-catheter management protocol can be implemented in other centres. Although most of the catheters were inserted by emergency physicians at the Rennes and Vitré hospitals the frequency of complications was similar to that encountered at the Lorient hospital where the protocol has been used for over ten years.

The one-year recurrence rate was $35.6 \%$, similar to published findings (17-49\%), including a recent metaanalysis that demonstrated a $32 \%$ PSP recurrence rate. ${ }^{8,9,15-17}$ We choose to manage as outpatient both PSP and SSP to reproduce our previous result and to show that this procedure is safe for both types of patients. Eventhough the recurrence rate seems higher among SSP patients, the management of the initial episode should not be different in our opinion.

The low complication rate is consistent with the findings of most published studies. The procedure gives rise to few complications even when larger chest tubes are used. ${ }^{18-20}$ Scars are almost invisible a few weeks after the catheter has been removed (unpublished data). The British and Belgian guidelines also propose needle aspiration (NA) as first-step treatment of PSP ${ }^{3,4}$ as several studies have shown this technique to be effective. ${ }^{21,22}$ A recent prospective randomised study that compared NA and chest tube drainage (CTD) reported that patients treated by NA stayed for significantly less time in hospital. ${ }^{12}$ However, in this study, $31 \%$ of the NA were hospitalized after up to 3 NA drainage attempts whereas our patients were immediately discharged after insertion of the pigtail catheter and a single chest X-ray was occasionally used to check catheter placement. NA and CTD were compared in two recent meta-analyses. ${ }^{23,24}$ Both studies acknowledged that NA is associated with a shorter stay 
in hospital but the Cochrane analysis concludes that CTD had a higher immediate success rate, although there was little supporting evidence. Walker and Maskell point out in their editorial that, while NA is associated with fewer bed days and adverse events, it has an initial risk of failure rate of $50 \% .^{25} \mathrm{We}$ believe that the use of pigtail catheters has features that make it more desirable than either chest tube or NA: a high success rate, ambulatory management and a low complication rate. This was also the conclusion reached by another recent meta-analysis that compared pigtail catheters and large-bore chest tubes. ${ }^{26}$

As underlined by Vuillard et al., a longer symptom onset seems to be associated with higher success rate of NA. ${ }^{27}$ Unfortunately, this information was not recorded during the study. It could be an interesting data, in order to guide physicians chosing the right technique for the right patient.

An Australian multicenter randomized controlled trial evaluated conservative and invasive management of PSP at first presentation. ${ }^{28}$ This trial provides modest evidence that conservative management of primary spontaneous pneumothorax was noninferior to interventional management, further studies are needed to assess this management, the main concern with this approach being patients safety.

Patients are being randomly assigned to either observation or needle aspiration and chest drain insertion. However, while observation alone is an interesting approach, ambulatory management with a pigtail catheter is reassuring for both patient and physician as the one-way valve prevents tension pneumothorax.

First-line pleurodesis for the initial management of spontaneous pneumothoraces has also been studied. Chen $e t$ al. proposed using minocycline pleurodesis after simple aspiration and drainage for the first episode of PSP to reduce the rate of recurrence. ${ }^{29}$ However, a prolonged length of stay (mean: 41 hours) in an emergency department is too long and this procedure is very painful (mean score of 8/10 on visual analog scale). ${ }^{29} \mathrm{We}$ do not know if this technique is still in use.

Another option is first line surgery. A recent randomised trial compared surgery and CTD for treating PSP. ${ }^{30}$ The authors concluded that first line surgery is an effective treatment with a significantly lower recurrence rate than CTD. This positive outcome is not surprising, but surgery is invasive and painful and has side effects, that were not studied. Moreover, it implies treating surgically patients who would never present with a second episode even without surgery, ie: $64.4 \%$ of our patients. First line surgery could be interesting if we could reliably identify patients likely to suffer a recurrence.

Our study has some limitations. First, the lack of a control group. The management of spontaneous pneumothoraces can and should be ambulatory whenever possible, the efficiency and safety small bore catheters with a one-way valve needed to be assessed before further study comparing this method with NA. Second, we treated relatively few cases of SSP, which makes it difficult to draw conclusions for these patients despite the fact that all cases of pneumothorax were managed using the same protocol in our centres. Only a few patients 
were lost to follow-up $(5 \cdot 4 \% ; \mathrm{n}=8)$. Perhaps our protocol may be difficult to implement in other centres since some health-care systems might not have the resources to monitor these patients so closely or to coordinate Emergency and Pulmonology departments. ${ }^{14}$ Nevertheless, our demonstration of the success of exclusive ambulatory management should motivate physicians to try it.

To conclude, we have shown that exclusive ambulatory management of spontaneous pneumothoraces with pigtail catheters and a one-way valve is efficient and safe, with a low rate of hospitalisation and no increase in one-year recurrence. Lastly the protocol can be implemented in other centres and emergency departments with similar results. Further randomised studies comparing needle aspiration and pigtail catheters could help determine which technique provides the best patient outcome. 
ACKNOWLEDGMENTS: The authors thank Dr Owen Parkes for editing the English text.

Alexandre Salé for literature search, data collection, writing, figures; Laurent Sohier for data collection, study design, data interpretation; Marine Campion for data analysis, figures; Régis Le Ho for data collection, corrections; Yann Bazin for data collection, literature search; Cedric Gangloff for data interpretation, corrections; Mallorie Kerjouan for data collection, corrections; Bertrand de Latour for data interpretation, corrections; Emmanuel Oger for study design, data analysis, data interpretation; and Stéphane Jouneau for writing, study design, data interpretation, figures and corrections. 


\section{REFERENCES}

1 Noppen M. Spontaneous pneumothorax: epidemiology, pathophysiology and cause. Eur Respir Rev Off J Eur Respir Soc 2010; 19: 217-9.

2 Bobbio A, Dechartres A, Bouam S, et al. Epidemiology of spontaneous pneumothorax: gender-related differences. Thorax 2015; 70: 653-8.

3 Henry M, Arnold T, Harvey J. BTS guidelines for the management of spontaneous pneumothorax. Thorax 2003; 58: ii39-52.

4 De Leyn P, Lismonde M, Ninane V, et al. Guidelines Belgian Society of Pneumology. Guidelines on the management of spontaneous pneumothorax. Acta Chir Belg 2005; 105: 265-7.

5 Baumann MH, Strange C, Heffner JE, et al. Management of spontaneous pneumothorax: an American College of Chest Physicians Delphi consensus statement. Chest 2001; 119: 590-602.

6 Tschopp J-M, Bintcliffe O, Astoul P, et al. ERS task force statement: diagnosis and treatment of primary spontaneous pneumothorax. Eur Respir J 2015; published online June 25.

DOI:10.1183/09031936.00219214.

$7 \quad$ Maskell NA. Pneumothorax management: time to improve the evidence base. Thorax 2017; 72: 1065-6.

8 Marquette C-H, Marx A, Leroy S, et al. Simplified stepwise management of primary spontaneous pneumothorax: a pilot study. Eur Respir J 2006; 27: 470-6.

9 Massongo M, Leroy S, Scherpereel A, et al. Outpatient management of primary spontaneous pneumothorax: a prospective study. Eur Respir J 2014; 43: 582-90.

10 Jouneau S, Sohier L, Desrues B. Ambulatory management of large primary spontaneous pneumothorax. Eur Respir J 2014; 43: 1215-1215.

11 Voisin F, Sohier L, Rochas Y, et al. Ambulatory management of large spontaneous pneumothorax with pigtail catheters. Ann Emerg Med 2014; 64: 222-8.

12 Thelle A, Gjerdevik M, SueChu M, Hagen OM, Bakke P. Randomised comparison of needle aspiration and chest tube drainage in spontaneous pneumothorax. Eur Respir J 2017; 49.

DOI:10.1183/13993003.01296-2016.

13 Brims FJH, Maskell NA. Ambulatory treatment in the management of pneumothorax: a systematic review of the literature. Thorax 2013; 68: 664-9.

14 Barrett TW, Schriger DL. Annals of Emergency Medicine Journal Club. Full of hot air? Do patients with large spontaneous pneumothoraces require hospitalization?: answers to the September 2014 Journal Club questions. Ann Emerg Med 2015; 65: 224-30.

15 Noppen M, Alexander P, Driesen P, Slabbynck H, Verstraeten A. Manual aspiration versus chest tube drainage in first episodes of primary spontaneous pneumothorax: a multicenter, prospective, randomized pilot study. Am J Respir Crit Care Med 2002; 165: 1240-4.

16 Chen J-S, Chan W-K, Yang P-C. Intrapleural minocycline pleurodesis for the treatment of primary spontaneous pneumothorax. Curr Opin Pulm Med 2014; 20: 371-6.

17 Walker S, Bibby A, Halford P, Stadon L, White P, Maskell N. Recurrence rates in primary spontaneous pneumothorax: a systematic review and meta-analysis. Eur Respir J 2018; published online July 12. DOI:10.1183/13993003.00864-2018.

18 Korczyński P, Górska K, Nasiłowski J, Chazan R, Krenke R. Comparison of Small Bore Catheter Aspiration and Chest Tube Drainage in the Management of Spontaneous Pneumothorax. Adv Exp Med Biol 2015; 866: 15-23. 
19 Contou D, Razazi K, Katsahian S, et al. Small-bore catheter versus chest tube drainage for pneumothorax. Am J Emerg Med 2012; 30: 1407-13.

20 Iepsen UW, Ringbæk T. Small-bore chest tubes seem to perform better than larger tubes in treatment of spontaneous pneumothorax. Dan Med J 2013; 60: A4644.

21 Ayed AK, Chandrasekaran C, Sukumar M. Aspiration versus tube drainage in primary spontaneous pneumothorax: a randomised study. Eur Respir J 2006; 27: 477-82.

22 Zehtabchi S, Rios CL. Management of emergency department patients with primary spontaneous pneumothorax: needle aspiration or tube thoracostomy? Ann Emerg Med 2008; 51: 91100, 100.e1.

23 Wang C, Lyu M, Zhou J, Liu Y, Ji Y. Chest tube drainage versus needle aspiration for primary spontaneous pneumothorax: which is better? J Thorac Dis 2017; 9: 4027-38.

24 Carson-Chahhoud KV, Wakai A, van Agteren JE, et al. Simple aspiration versus intercostal tube drainage for primary spontaneous pneumothorax in adults. Cochrane Database Syst Rev 2017; published online Sept 7. DOI:10.1002/14651858.CD004479.pub3.

25 Walker SP, Maskell N. Pneumothorax management—chest drain or needle aspiration? J Thorac Dis 2017; 9: 3463-4.

26 Chang S-H, Kang Y-N, Chiu H-Y, Chiu Y-H. A Systematic Review and Meta-Analysis Comparing Pigtail Catheter and Chest Tube as the Initial Treatment for Pneumothorax. Chest 2018; published online Feb. DOI:10.1016/j.chest.2018.01.048.

27 Vuillard C, Dib F, Achamlal J, et al. Longer symptom onset to aspiration time predicts success of needle aspiration in primary spontaneous pneumothorax. Thorax 2019; 74: 780-6.

28 Brown SGA, Ball EL, Perrin K, et al. Conservative versus Interventional Treatment for Spontaneous Pneumothorax. N Engl J Med 2020; 382: 405-15.

29 Jouneau S, Sohier L, Desrues B. Pleurodesis for primary spontaneous pneumothorax. Lancet Lond Engl 2013; 382: 203.

30 Olesen WH, Katballe N, Sindby JE, et al. Surgical treatment versus conventional chest tube drainage in primary spontaneous pneumothorax: a randomized controlled trial $\uparrow$. Eur J Cardiothorac Surg 2018; published online March 2. DOI:10.1093/ejcts/ezy003. 
Table 1: main patient characteristics

\begin{tabular}{|c|c|c|c|}
\hline Variables & $\begin{array}{c}\text { All pneumothoraces } \\
(\mathbf{N}=\mathbf{1 4 8})\end{array}$ & $\begin{array}{c}\text { PSP } \\
(\mathbf{N}=\mathbf{1 2 9})\end{array}$ & $\begin{array}{c}\text { SSP } \\
(\mathbf{N}=19)\end{array}$ \\
\hline $\begin{array}{c}\text { Age (years) } \\
\text { mean (SD) }\end{array}$ & $31.0(13)$ & $27.3(8.3)$ & $56.6(9.6)$ \\
\hline $\begin{array}{c}\text { Male } \\
\mathbf{N}(\%)\end{array}$ & $110(74.3)$ & $97(75.2)$ & $13(68.4)$ \\
\hline $\begin{array}{c}\text { Smokers } \\
\mathbf{N}(\%)\end{array}$ & $113(76.3)$ & $95(73.6)$ & $18(94.7)$ \\
\hline $\begin{array}{c}\text { Smoking history (pack- } \\
\text { years) } \\
\text { mean (SD) }\end{array}$ & $11.8(11.6)$ & $7.8(6.9)$ & $31.5(9.3)$ \\
\hline $\begin{array}{c}\text { Body mass index (kg.m } \\
\text { 2)mean (SD) }\end{array}$ & $20.8(2.3)$ & $20.4(2.1)$ & $23.4(2.3)$ \\
\hline $\begin{array}{c}\text { Previous pneumothorax } \\
\mathbf{N}(\%)\end{array}$ & $22(14.9)$ & $18(13.9)$ & $4(21.1)$ \\
\hline $\begin{array}{c}\text { Right-side pneumothorax } \\
\mathbf{N}(\%)\end{array}$ & $101(68.2)$ & $90(69.8)$ & $11(57.9)$ \\
\hline
\end{tabular}

PSP =Primary spontaneous pneumothorax;

SSP = Secondary spontaneous pneumothorax;

$\mathrm{SD}=$ standard deviation

Table 2: Success rates for different types of pneumothorax

\begin{tabular}{|c|c|c|c|c|}
\hline Day of success & $\begin{array}{l}\text { Day } 2 \\
\text { N }(\%)\end{array}$ & $\begin{array}{l}\text { Day } 4 \\
\text { N }(\%)\end{array}$ & $\begin{array}{l}\text { Day } 6 \\
\text { N (\%) }\end{array}$ & $\begin{array}{c}\text { Pleurodesis } \\
\text { N }(\%)\end{array}$ \\
\hline \multicolumn{5}{|l|}{ PSP } \\
\hline All $(\mathrm{N}=129)$ & $59(45.7)$ & $55(42.6)$ & $9(6.9)$ & $6(4.6)$ \\
\hline First episode $(\mathrm{N}=111)$ & $51(46.0)$ & $48(43.2)$ & $8(7.2)$ & $4(3.6)$ \\
\hline Recurrence $(\mathrm{N}=18)$ & $8(44.4)$ & $7(3.9)$ & $1(5.5)$ & $2(11.1)$ \\
\hline \multicolumn{5}{|l|}{ SSP } \\
\hline All $(\mathbf{N}=19)$ & $6(31.5)$ & $9(47.3)$ & $1(5.2)$ & $3(15.7)$ \\
\hline First episode $(\mathrm{N}=15)$ & $4(26.7)$ & $8(53.3)$ & $1(6.7)$ & $2(13.3)$ \\
\hline Recurrrence $(\mathrm{N}=4)$ & $2(50.0)$ & $1(25.0)$ & 0 & $1(25.0)$ \\
\hline Total & $65(43.9)$ & $64(43.2)$ & $10(6.7)$ & $9(6.1)$ \\
\hline
\end{tabular}

PSP = Primary spontaneous pneumothorax;

$\mathrm{SSP}=$ Secondary spontaneous pneumothorax

Figure 1: Pigtail catheter and one way valve in position

Figure 2: Ambulatory management of large spontaneous pneumothoraces, adapted from Voisin et al ${ }^{11}$

$C X R=$ Chest $X$-ray; Success $=$ Complete or nearly complete lung re-expansion

Figure 3: Flow chart of patient evolution. In bold, number of patient exclusively ambulatory managed $(\mathrm{N}=122$, $82.4 \%)$.

$P S P=$ Primary spontaneous pneumothorax $;$ SSP = Secondary spontaneous pneumothorax

Figure 4: Kaplan Meyer survival curves for primary spontaneous pneumothorax (PSP) and secondary spontaneous pneumothorax (SSP) with 95\% Hall-Wellner bands. 


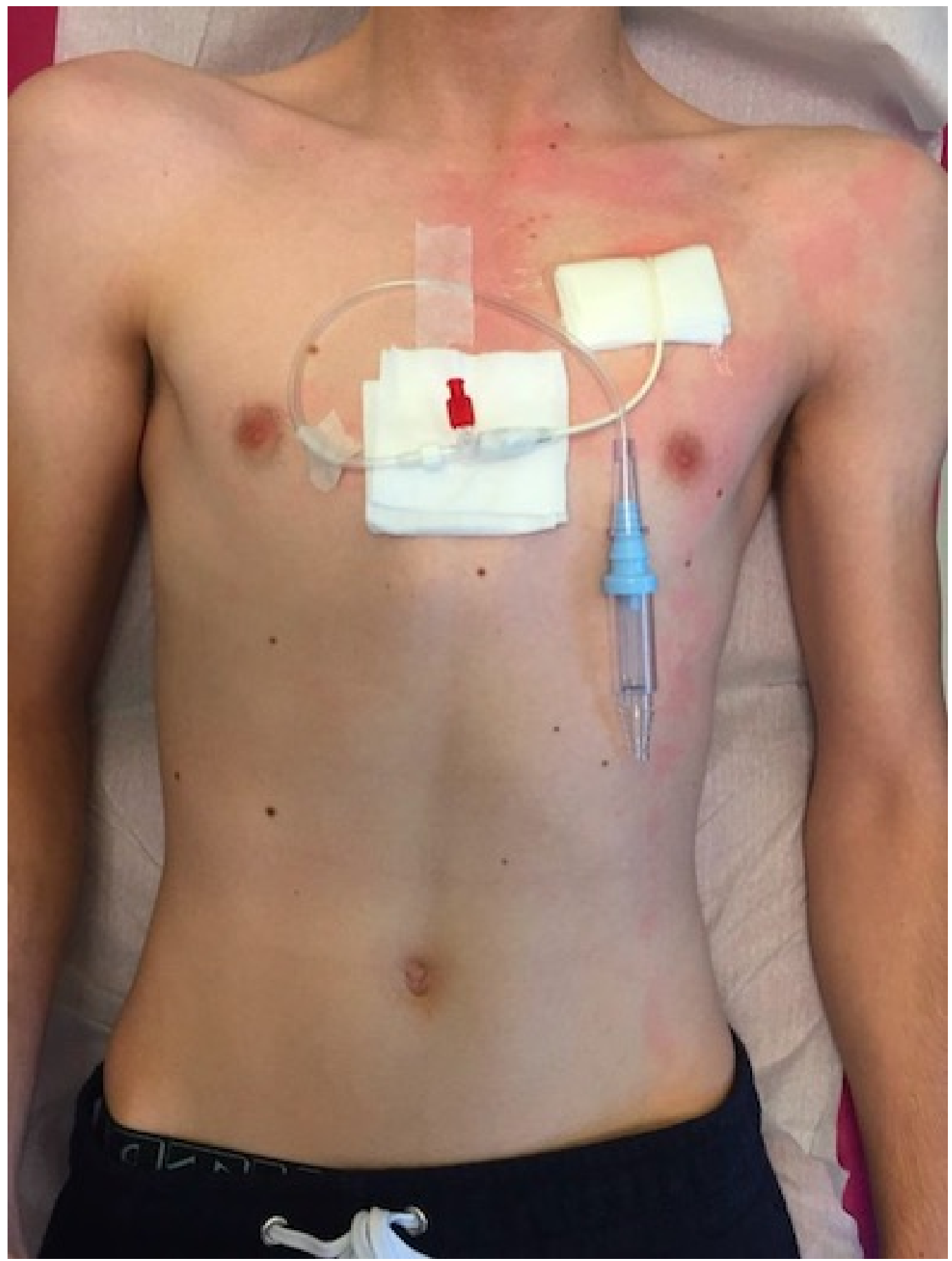




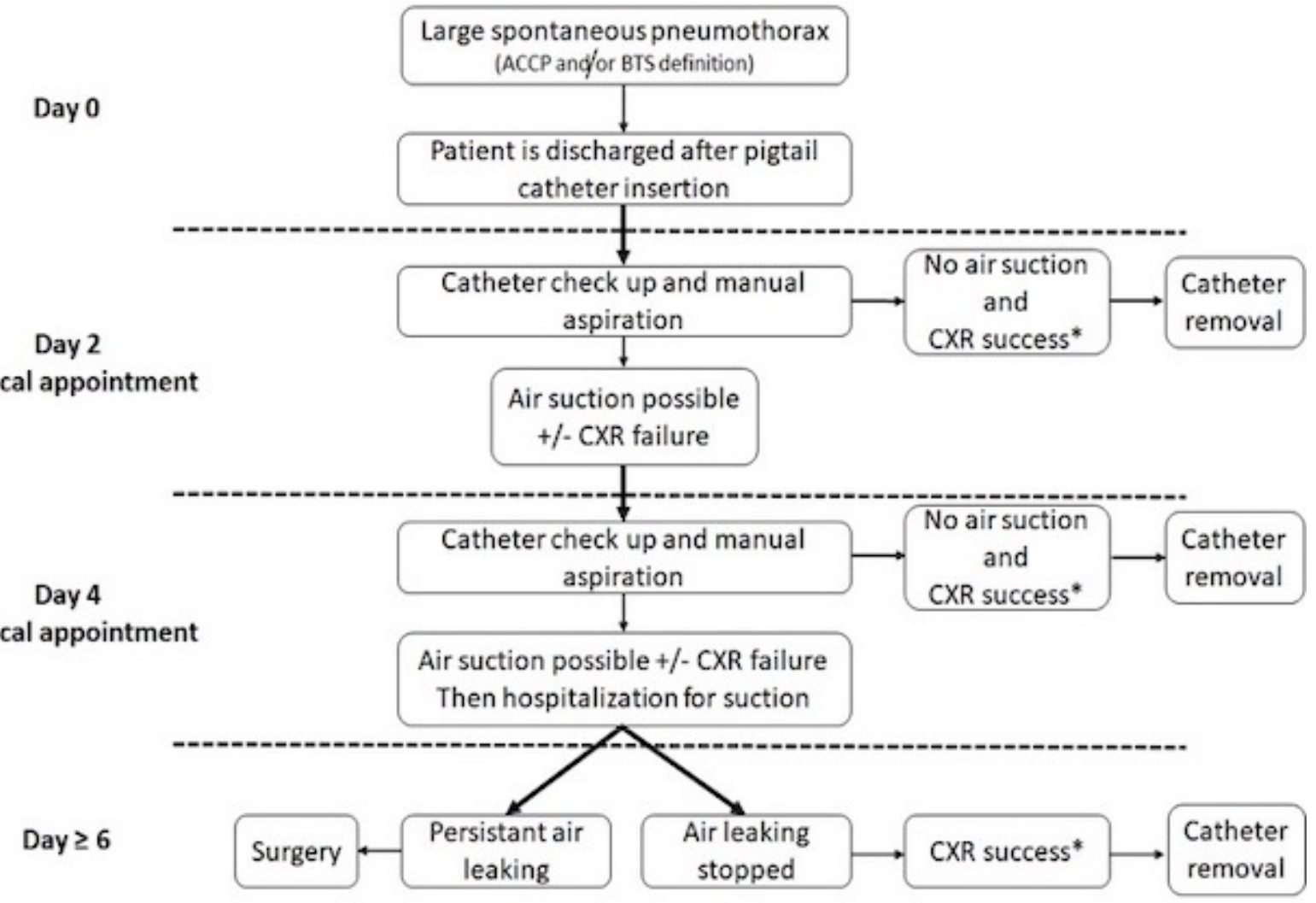




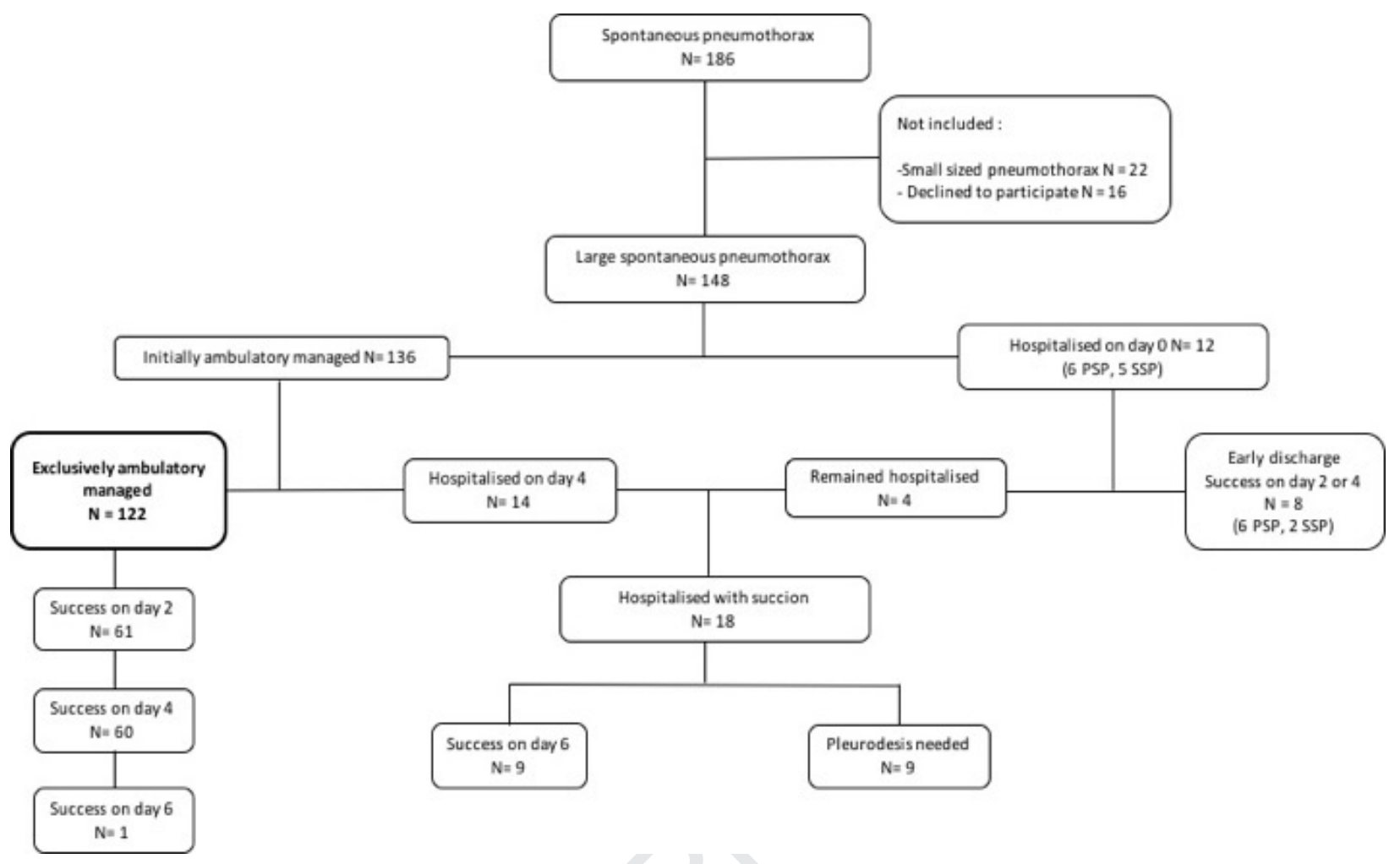




\section{Recurrence free survival}

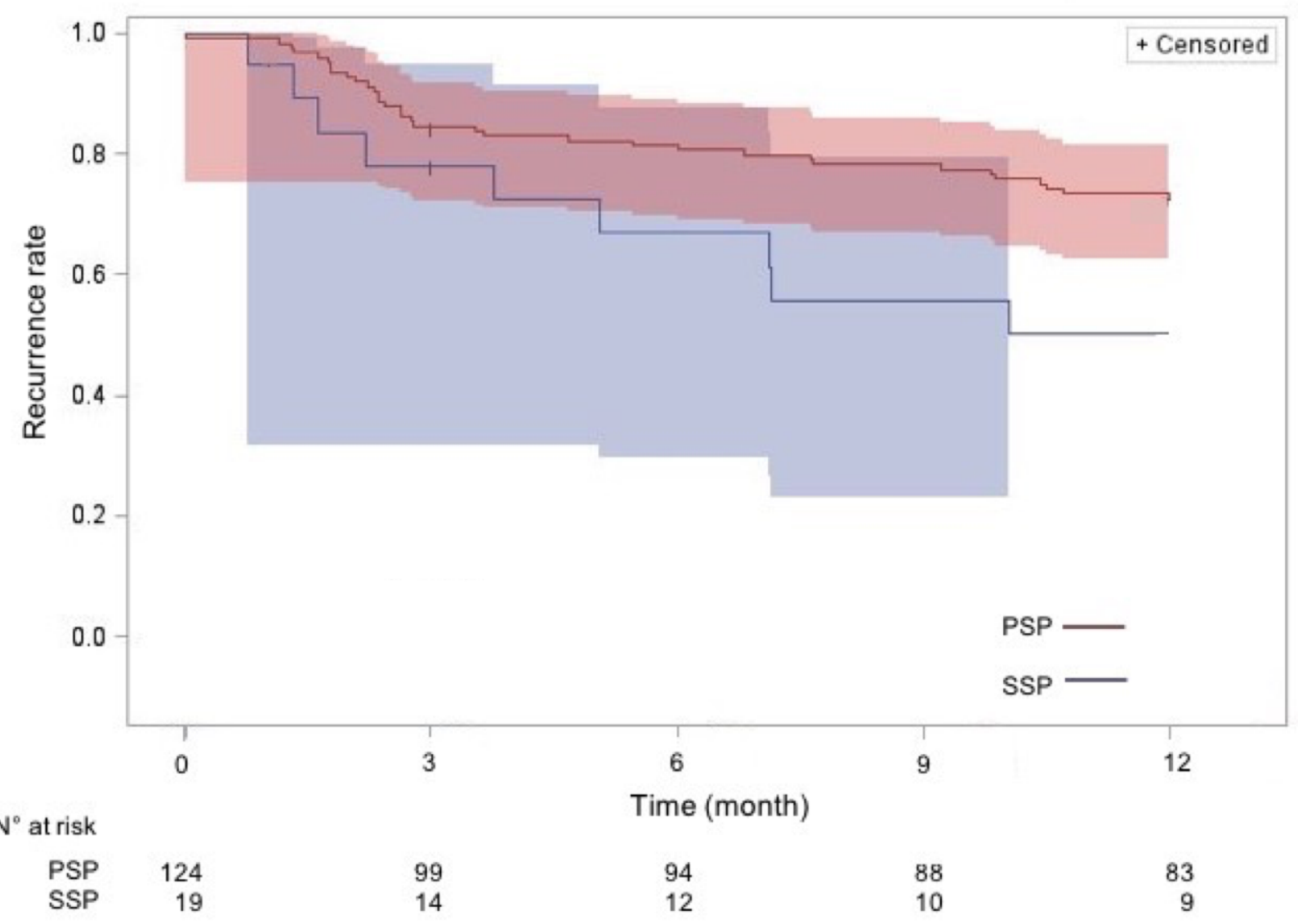




\section{HIGHLIGHTS}

Ambulatory management of spontaneous pneumothoraces is possible with pigtail catheters.

Ambulatory management of spontaneous pneumothoraces is safe and efficient.

Ambulatory anagement of spontaneous pneumothoraces can be impletementd in new centers. 


\section{Declaration of interests}

The authors declare that they have no known competing financial interests or personal relationships that could have appeared to influence the work reported in this paper.

$\bigotimes$ The authors declare the following financial interests/personal relationships which may be considered as potential competing interests:

Dr. Salé reports personal fees and non-financial support from AIRB, Boehringer, Roche, outside the submitted work; Dr. de Latour has nothing to disclose; Dr. Sohier has nothing to disclose; Dr. Campion has nothing to disclose; Dr. Le Ho has nothing to disclose; Dr. Bazin has nothing to disclose; Dr. Gangloff has nothing to disclose; Dr. Kerjouan has nothing to disclose; Dr. Oger has nothing to disclose; Dr. Jouneau reports grants from Rennes University Hospital (COREC 2011 funding), the 'Association pour les insuffisants respiratoires de Bretagne' (AIRB), the French Ministry of Labour (DIRRECTE), and Novartis Pharma., during the conduct of the study; grants and non-financial support from AIRB, Boehringer, Gilead, GSK, LVL, Roche, Savara / Serendex, personal fees and non-financial support from Actelion, AIRB, Astra Zeneca, BMS, Boehringer, Chiesi, GSK, Mundipharma, Novartis, Pfizer, Roche, outside the submitted work. 